\title{
Double Jab: Survey Evidence on Vaccine Hesitancy, Beliefs, and Attitudes in India
}

Anirudh Tagat ${ }^{1}$, Hansika Kapoor ${ }^{2,3}$, Varun Arora ${ }^{4}$, Sujoy Chakravarty ${ }^{4,5}$, Shagata Mukherjee ${ }^{6}$, and

\author{
Shubhabrata Roy ${ }^{4}$ \\ ${ }^{1}$ Department of Economics, Monk Prayogshala \\ ${ }^{2}$ Department of Psychology, Monk Prayogshala \\ ${ }^{3}$ Neag School of Education, University of Connecticut \\ ${ }^{4}$ Behavioral Insights Architecture and Strategy (BIAS) Inc. \\ ${ }^{5}$ Centre for Economic Studies and Planning, Jawaharlal Nehru University \\ ${ }^{6}$ Centre for Social and Behaviour Change, Ashoka University
}

\begin{abstract}
Hansika Kapoor 0000-0002-0805-7752
Anirudh Tagat $\underline{0000-0002-7707-453 X}$

Shagata Mukherjee 0000-0003-0067-8690
\end{abstract}

Author Note

The authors are grateful to two anonymous referees and senior editor Xiaoli Nan for helpful comments and suggestions. We thank Madhumeta Rajkumar and Subhashish Sarkar for valuable help with data collection. This research was funded by BIAS Inc., New Delhi. The authors declare that there are no potential conflicts of interest with respect to the research, authorship, and/or publication of this article. Correspondence concerning this article should be addressed to Anirudh Tagat, Research Author, Department of Economics, Monk Prayogshala (Sec 25), 4114, Oberoi Garden Estates, Near Chandivali Studios, Powai, Mumbai-400072, India.

Email: at@monkprayogshala.in

This is a pre-print version of the publication: Tagat, A., Kapoor, H., Arora, V., Chakravarty, S., Mukherjee, S., \& Roy, S. (2022). Double Jab: Survey Evidence on Vaccine Hesitancy, Beliefs, and Attitudes in India. Health Communication, 1-12. 


\begin{abstract}
India witnessed a large surge in COVID-19 cases in April 2021, a second wave of nearly 350,000 daily new infections across the country. As of December 2021, cases have reduced drastically, in part due to greater vaccine coverage across the country. This study reports results on vaccine hesitancy, attitudes, and behaviors from an online survey conducted between February and March 2021 in nine Indian cities $(\mathrm{N}=518)$. We find that vaccine hesitancy negatively predicts willingness to take the vaccine, and beliefs about vaccine effectiveness supersede hesitancy in explaining vaccine uptake. Furthermore, we find that mask-wearing and handwashing beliefs, information sources related to COVID-19, and past COVID-19 infection and testing status are all strongly associated with the hypothetical choice of vaccine. We discuss these findings in the context of behavioral theories as well as outline implications for vaccinerelated health communication in India.
\end{abstract}

Keywords: COVID-19 vaccine, health communication, preventive health behaviors, vaccine hesitancy, vaccine nationalism 


\section{Double Jab: Survey Evidence on Vaccine Hesitancy, Beliefs, and Attitudes in India}

Between April and August 2021, India experienced a second wave of COVID-19 infections, topping the global record for highest number of daily cases (nearly 300,000 on average). This is nearly 3 times higher than the comparable case load during the first wave (September - December 2020), indicating that health systems in India are under unprecedented pressure in treating patients infected with COVID-19. Although there is no robust evidence on factors leading up to the recent surge of cases, there are both individual and state-related factors that could explain the second wave of cases in India (Asrani et al., 2021; Thiagarajan, 2021b).

A key development distinguishing the recent second wave of cases from the first wave also has to do with vaccine availability to more than 250 million Indians aged 45 years and above. It is possible that availability of a vaccine has reduced perceived risk associated with COVID-19. For example, Andersson et al. (2021) showed that anticipation of a COVID-19 vaccine sharply reduced social distancing and willingness to stay at home in Sweden. Other studies have suggested that risky behavior is often observed following medical innovation, for example in the case of HIV treatment (Chan et al., 2016). There is also extensive work on the benefits of vaccination (not specific to COVID-19) that indicate they help in achieving herd immunity (Tate et al., 2019), and have various socio-economic benefits in terms of fewer losses due to illness (Bloom et al., 2018). Thus, it becomes important to examine how the development of vaccines has impacted different attitudes, perceptions, and behaviors related to COVID-19 (both prior to and after vaccination). This is more so the case in a developing country context such as India, given the scale at which vaccination needs to take place to achieve immunity against COVID-19 infection and prevent overwhelming the health system. 


\section{COVID-19 Vaccination in India}

The Government of India, led by Prime Minister Narendra Modi, launched a nationwide vaccination campaign on January 16, 2021, following clinical trials and authorization of two locally-produced vaccines: the AstraZeneca-Oxford University vaccine (Covishield, or ChAdOx1 nCoV-19) and the indigenous Bharat Biotech vaccine (Covaxin). Although there have been concerns around clinical trials of Covaxin (Bhuyan, 2021), it has been administered widely and since reported efficacy against novel variants of the coronavirus (Sapkal et al., 2021). Given the challenge of inoculating more than 1.3 billion people in India, this first phase prioritized healthcare workers (frontline workers and medical staff at hospitals), and was followed by availability to individuals aged above 60 years and those above 45 years of age with comorbidities starting March 1, 2021. Vaccines were made available to the public at privatelyowned and public hospitals as well as specialized COVID-19 vaccination centers. By March 15 2021, nearly 35 million doses (first or second) of Covaxin and Covishield had been administered (COVID-19 India Dashboard, 2021).

The vaccination was made available to all individuals aged above 45 years starting April 12021 , and coincided with overall confirmed cases in India at 12.3 million. In the next phase of the vaccination drive, the Government of India allowed all those above 18 years of age to get vaccinated starting May 1 2021. There have also been recent efforts to approve additional vaccines (such as Sputnik V and Moderna) to help meet the potential demand for vaccination in India. With the exception of Goruntla et al. (2021) and Kumari et al. (2021), however, there is virtually no information on attitudes, perceptions, and beliefs related to the COVID-19 vaccine in India. This is particularly pressing given the scale at which vaccination is proposed in India as well as past indications of low adult vaccination rates in India (Dash et al., 2020). Although 
multi-country studies such as Lazarus et al. (2021) include India, they were typically conducted much earlier (June 2020) and also do not offer any country-specific insights that could prove useful in developing policies on health and vaccine-related messaging in India.

\section{Vaccine Hesitancy}

Scholarship on COVID-19 vaccine acceptance and rates of vaccine hesitancy has been accumulating since 2020. In a systematic review by Sallam (2021), 31 papers published between February and December 2020 were considered, providing large variations in vaccine acceptance rates among the general population (ranging from $23.6 \%$ in Kuwait to $97 \%$ in Ecuador).

Similarly, Lazarus et al. (2021) identified a potential acceptance rate of $71.4 \%$ among participants from 19 countries, including India. However, research has also suggested that as the pandemic has progressed over time, the intention to get vaccinated has reduced (Robinson et al., 2021). Further, COVID-19 vaccine hesitancy is related to specific attitudes and beliefs, ranging from believing in the artificial origin of the virus (Salali \& Uysal, 2020), lower perceived severity of COVID-19 (Schwarzinger et al., 2021), distrust in vaccines and mistrust in government (Jennings et al., 2021), as well as exposure to misinformation (Loomba et al., 2021). In the Indian context, research has identified qualitative views regarding the COVID-19 vaccine among diverse groups such as doctors and housewives (Kumari et al., 2021) and examined preliminary predictors of vaccine acceptance (Goruntla et al., 2021).

As wide-scale vaccination is critical for managing the spread of COVID-19 and thereby controlling the pandemic, it is important to understand attitudes, beliefs, and behaviors contributing to vaccine hesitancy and resistance (typically prior to getting vaccinated). Increasing confidence in vaccines in the long-term and appealing to prosocial motivations to take up the vaccine (counteracting negative emotions associated with misinformation and mistrust; Bavel et 
al., 2020) are some strategies to facilitate evidence-based vaccination efforts (Chou \& Budenz, 2020). Similarly, using behavioral interventions to make vaccines more accessible and contribute toward developing the norm of getting vaccinated are also recommended to boost vaccination rates (Chevallier et al., 2021).

\section{The Present Study}

The present investigation examines variables associated with vaccine uptake, perceived effectiveness of preventive health behaviors, and beliefs about the likelihood of COVID-19 infection. As of October 2021, there are a few studies in India that have looked at vaccine hesitancy based on attitudinal surveys, either at the community (Danabal et al., 2021) or individual level (Chowdhury et al., 2021). This study contributes to emerging evidence on factors associated with vaccine hesitancy in India, even as overall fully vaccinated levels remain low (38\% as of December 2021). Targeting these factors will be critical for developing communication policies in India going forward (Kanabar \& Bhatt, 2021). Against the background of scholarship associated with beliefs, behaviors, and the COVID-19 vaccine (e.g., Murphy et al., 2021), the present study aimed to explore the attitudes associated with the COVID-19 vaccine among Indian participants. The theory of planned behavior (Ajzen, 1991) was used to contextualize the potential influence of attitudes and beliefs in contributing to behaviors regarding the vaccine. Specifically, the following research questions were posed: RQ1: What are the attitudinal and informational factors associated with vaccine uptake among Indians?

RQ2: What are the attitudinal and informational factors associated with hypothetical vaccine choice ${ }^{1}$ among Indians?

\footnotetext{
${ }^{1}$ Data were collected in India during February and March, 2021, when a significant proportion of the population was still ineligible to receive any COVID-19 vaccine. Therefore, this was framed as a hypothetical vaccine choice to
} 


\section{Method}

\section{Participants and Procedure}

The sample $(N=518)$ was drawn using exponential non-discriminatory snowball sampling methods in nine cities (Bangalore, Chandigarh, Chennai, Gurugram, Hyderabad, Indore, Mumbai, New Delhi, and Kolkata) between 3 February and 27 March 2021. The project was approved by the institutional review board at Monk Prayogshala prior to data collection (\#047-020). All respondents were recruited via multiple-site entry methods (Reips, 2002), and field recruiters in each city were incentivized to share survey links to boost participation rate. Participants responded to the survey in English and provided written consent prior to taking the survey. In all, approximately 1500 individuals received the survey link, of which 518 responded. Of these, non-missing data on socio-demographic variables as well as other questions were available only for 375 . Nearly $75 \%$ of respondents were currently residing in the nine cities mentioned above, the remainder reporting current residences in smaller towns in the same state. Approximately $52 \%$ of the sample identified as women, and nearly $88 \%$ reported having completed at least an undergraduate degree at the time of the survey. The majority identified themselves as Hindu $(N=353)$. Approximately $17 \%$ of the sample were students, and $41 \%$ reported being employed in private organizations, with others either employed in government organizations or self-employed (25\%). Descriptive statistics are presented in Table A.1 in the online appendix.

\section{Measures}

\section{COVID-19 Attitudinal and Informational Factors}

enable future predictions of real vaccine choices. This enables us to understand drivers of vaccine choice once the participants in our sample became eligible to be vaccinated. 
These included data on perceived effectiveness of mask-wearing, handwashing, and vaccination in preventing COVID-19 infection (World Health Organization, 2020). Participants rated these on a 100 -point scale $(0=$ not at all effective to $100=$ extremely effective $)$. Additionally, participants responded to whether they follow strict social distancing, or if they followed it only with certain groups of individuals (e.g., family members, friends, strangers). The most preferred source of COVID-19-related information was also included, with the following options: newspapers, internet, social media, friends and family, or neighbors. Finally, a measure of perceived disease threat from COVID-19 was also included: "How likely do you think you are to contract COVID-19?" (0 = not at all to $100=$ extremely likely).

Vaccine Hesitancy Index. A composite vaccine hesitancy measure was constructed using cumulative responses to three questions on vaccine characteristics: ingredients of the vaccine, side effects caused by the vaccine, and the cost of the vaccine (similar to Wagner et al., 2019). Participants responded to whether or not they were concerned about each of these using a 100 -point scale $(0=$ not at all concerned to $100=$ extremely concerned $)$. The index was reliable and internally consistent, Cronbach's $\alpha=0.69$.

\section{Socio-demographic Factors}

Where available, additional socio-demographic factors such as age (in years), religion, caste, annual post-tax household income (categories between INR 500,000 or less to INR 4 million or more), education (categories), occupational status (categories), and health status (whether or not they suffered from a long-term health condition) were also collected.

\section{Confirmed Cases and Vaccines Administered}

Data on locality was matched with secondary data on caseload as well as vaccine doses administered during the survey period of 3 February to 27 March 2021. This data was retrieved 
from the COVID-19 India Dashboard (2021), which contains district-level data on cases, vaccination status, among others.

\section{Dependent Variables}

The main dependent variables were vaccine uptake and hypothetical vaccine choice. To measure vaccine uptake, respondents were asked "How likely are you to take any COVID-19 vaccine?" on a 100 -point scale $(0=$ not at all to $100=$ very likely $)$. Next, the measures of vaccine choice were derived from the response to the question "If you faced a choice between a free Government vaccine and a foreign vaccine produced by a private company which costs INR 1000 per head, which one would you choose?", where the responses were 1 = surely take Indian vaccine, 2 = surely take foreign vaccine, $3=$ depends on relative effectiveness, $4=$ would take foreign one if it was given for free, and $5=$ neither, I don't believe it will make a difference. Binary variables were derived from respondents that chose either (1) or (3), where the first variable took a value of 1 if they indicated that they preferred an Indian vaccine (and zero otherwise), and the second variable took a value of 1 if they indicated that their choice of vaccine depended on its relative effectiveness, and zero otherwise.

\section{Model Development}

An empirical model was developed hypothesizing a relationship between the measure of vaccine hesitancy and vaccine uptake. Additional covariates included in the model were the beliefs about mask-wearing, handwashing, vaccine effectiveness; practicing social distancing, perceived COVID-19 disease threat, sources of information related to COVID-19, and infection status. Location-matched averages over the survey period for COVID-19 caseload and number of vaccine doses administered were also used. Ordinary least squares regression was used for testing the factors associated with vaccine uptake and perceived vaccine efficacy. To examine 
the role of beliefs around effectiveness in addition to other vaccine-related beliefs, two models are estimated: one without belief on COVID-19 vaccine effectiveness, and one with this variable. Logistic regression models were used for testing hypothetical preference for a free Indian vaccine and vaccine choice depending on relative efficacy of available vaccines. All regressions used heteroskedasticity-adjusted robust standard errors, and odds ratios are reported for results from logistic regression models.

\section{Results}

\section{Factors affecting vaccine uptake}

Figures $1 \mathrm{a}$ and $1 \mathrm{~b}$ plot the OLS regression coefficients of the key explanatory variables on vaccine uptake (RQ1). The regression table is presented in the online appendix (Table A.2, Column 2). The left panel contains the results of the regression without including beliefs related to vaccine efficacy, whereas the right panel includes this variable in addition to the vaccine hesitancy index, beliefs regarding mask-wearing, handwashing, and social distancing (Table A.2, Column 3). Figure $1 \mathrm{~b}$ shows the coefficients from the same two regression models, including effects for COVID-19 information sources as well as COVID-19 infection status. These figures suggest that a higher score on the vaccine hesitancy index is associated with lower willingness to take any COVID-19 vaccine $(B=-0.039 ; p<0.1)$ in the model without belief regarding vaccination efficacy. Positive attitudes toward mask-wearing and handwashing result in a higher stated vaccine uptake $\left(B_{\text {mask }}=0.30\right.$ and $\left.B_{\text {handwash }}=0.23 ; p<0.01\right)$. In contrast, when beliefs regarding vaccine efficacy are included in the model, both vaccine hesitancy as well as beliefs regarding handwashing efficacy in preventing COVID-19 are no longer statistically significant. Having a stronger belief that vaccines are effective was positively associated with vaccine uptake 
$(B=0.71, p<0.01)$, whereas beliefs related to efficacy of mask-wearing continued to have a statistically significant and positive association with vaccine uptake $(B=0.15, p<0.01)$. In both cases, perceived COVID-19 disease threat did not significantly explain vaccine uptake. Notably, as seen in Figure 1b, potentially being previously infected (but not having been tested) strongly reduces vaccine uptake in our sample $(B=-9.3, p<0.1)$, suggesting that availability of testing, beliefs regarding testing, or beliefs regarding pre-existing antibodies could also be associated with willingness to take the vaccine. Further, no social distancing with family, friends, and neighbors increased the likelihood of vaccine uptake $(B=12.65, p<.10)$.

\section{Factors affecting hypothetical choice of vaccine}

Figures $2 \mathrm{a}$ and $2 \mathrm{~b}$ plot odds ratios from logistic regressions of choice of the Indian vaccine and respondents that indicated whether relative efficacy mattered for their choice of vaccine (RQ2). These results can also be found in Table A.2 (Columns 5 and 7, respectively). A higher score on the vaccine hesitancy index lowered the odds of taking the (hypothetically free) Indian vaccine $(\mathrm{OR}=0.996, p<0.05)$, whereas it raised the odds of citing relative efficacy as a determining factor for choice of vaccine $(\mathrm{OR}=1.005, p<0.05)$. A one unit increase in the belief that vaccines were effective in preventing COVID-19 infection increased the odds of taking the Indian vaccine $(\mathrm{OR}=1.02, p<0.01)$. Mask-wearing beliefs reduced the odds of citing relative efficacy $(\mathrm{OR}=0.987, p<0.1)$. In contrast, positive attitudes towards handwashing in reducing COVID-19 spread was associated with significantly higher odds of citing relative vaccine efficacy in vaccine choice $(\mathrm{OR}=1.018, p<0.05)$.

Importantly, the source of information on COVID-19 appears to significantly affect the odds of choice of the Indian vaccine, as well as citing relative efficacy in determining vaccine 
choice. Those who mainly received COVID-19-related information from the internet were far less likely to take the Indian vaccine $(\mathrm{OR}=0.24, p<0.01)$, but more likely to cite relative efficacy in driving choices $(\mathrm{OR}=3.62, p<0.05)$, relative to the case of individuals who primarily sought information from their neighbors. Similarly, those who accessed COVID-19 information mainly from newspapers and magazines increased their odds of choosing on the basis of relative efficacy $(\mathrm{OR}=3.05, p<0.05)$ and reduced their odds of choosing the Indian vaccine $(\mathrm{OR}=0.43, p<0.05)$. In addition, individuals who were previously not infected and not tested had lower odds of choosing the Indian vaccine $(\mathrm{OR}=0.59, p<0.10)$, but had higher odds of choosing a vaccine based on relative efficacy $(\mathrm{OR}=2.15, p<0.05)$.

Not many socio-demographic variables appeared to determine vaccine uptake nor hypothetical vaccine choice. A notable exception was age, where older individuals increased their odds of choosing the Indian vaccine $(\mathrm{OR}=1.28, p<0.1)$ and reduced their odds of citing relative efficacy for choosing a vaccine $(\mathrm{OR}=0.54, p<0.01)$, though this effect was not linear (since the term on squared-age showed the opposite signs). On average, respondents in higher self-reported post-tax household income categories (e.g., INR 2 to 4 mn annually) reported that their choice of vaccine would depend on relative efficacy $\left(\mathrm{OR}_{20-40 \mathrm{~L}}=3.51, p<0.05\right)$.

\section{Discussion}

As opposed to 2020, current COVID-19 data includes both number of cases as well as number of vaccination doses administered. Despite the development and rollout of multiple COVID-19 vaccines, a certain proportion of the world's population remains hesitant to receive any vaccine (e.g., Lazarus et al., 2021), thereby influencing global public health efforts to combat the pandemic. Further, the extent of COVID-19 vaccine hesitancy varies across nations, 
with India tending toward a relatively high acceptance rate of over 70\% as of June 2020 (Lazarus et al., 2021). To gain additional insight into the factors associated with vaccine uptake (RQ1) and hypothetical vaccine choice (RQ2), we investigated how attitudinal and informational factors influence these behaviors.

Results suggested that people who believed the effectiveness of health behaviors like hand-washing prior to taking the vaccine were more likely to take the vaccine as well—perhaps as vaccination is the gold standard of preventive health behaviors and is often the key medical intervention needed to prevent infection. On the other hand, when individuals held concerns about the vaccine, such as those related to its cost, they were less likely to opt for vaccination. Even when making a hypothetical choice between a free Indian vaccine and one based on relative efficacy between an Indian and foreign one, beliefs about vaccine hesitancy, general vaccine effectiveness, as well as the source of information influenced the choice.

\section{Factors affecting vaccine uptake}

In general, results indicated that beliefs in effectiveness of preventive health behaviors (prior to vaccination) increased the likelihood of vaccine uptake, whereas concerns about vaccine cost, ingredients, and side effects reduced the same (RQ1). Positive attitudes toward maskwearing emerged as a robust predictor of the decision to get vaccinated, in models with and without beliefs about COVID-19 vaccine effectiveness. On the other hand, favorable attitudes toward handwashing associated with higher vaccine uptake did not hold when beliefs about COVID-19 vaccine effectiveness were included. The knowledge that COVID-19 transmission is primarily airborne is likely to have influenced these beliefs, and perhaps mask-wearing was considered to be a more direct way of preventing such transmission. Such a belief would broadly adhere to the health belief model (HBM), which indicates that mask-wearing and taking a 
vaccine is more effective against COVID-19, as opposed to hand-washing (Tong et al., 2020).

The lack of association between beliefs on handwashing and vaccine uptake once beliefs on vaccine efficacy are included could also be explained by lack of access to adequate handwashing facilities (Brauer et al., 2020; Ray, 2020). Among attitudes toward the effectiveness of preventive health behaviors, the belief that COVID-19 vaccines (not any specific one) are effective was the strongest predictor of vaccine uptake. In line with the theory of planned behavior, attitudes toward the utility of vaccines, as well as other health behaviors, contributed to future vaccination behavior (see also Schmid et al., 2017). Behaviors pertaining to social distancing also predicted intention to get vaccinated; specifically, if individuals were not practicing any social distancing with family, friends, and neighbors, they were more likely to get vaccinated. It is likely that such individuals perceived the interchangeable nature of one preventive health behavior with another, and may have justified not practicing social distancing with their intent to get vaccinated (see also Andersson et al., 2021). It is also possible that there is a lack of knowledge on appropriate social distancing in the Indian context (Gudi et al., 2020).

Of interest was the negative effect of having been potentially infected but not tested on vaccine uptake. Indians who had assumed that they were COVID-19 positive, but had not been tested for various reasons (stigma, inconvenience, testing hesitancy due to nature of test, limited testing outreach and capacity) were less likely to take the vaccine. Perhaps such individuals do not have a clear reason for getting vaccinated, based on the assumption that they may already have COVID-19 antibodies. Misconceptions related to gaining immunity (e.g. "Indians have natural immunity from COVID-19”) from the virus may also be driving this result (Sahoo et al., 2020). Again, when beliefs about the effectiveness of COVID-19 vaccines were included in the model, this effect was null. The primary source of COVID-19 information also did not impact 
vaccine uptake; disaggregating news sources as well as nature of information consumed/beliefs in the veracity of the same can be investigated in future research.

\section{Factors affecting hypothetical choice of vaccine}

In terms of vaccine choice between a hypothetically free Indian vaccine and stating that choice depended on relative efficacy between an Indian and foreign vaccine, significant attitudinal and informational predictors were nearly a mirror image of each other (RQ2). This was due to the two dependent variables associated with vaccine choice accounting for $85 \%$ of all responses. Greater hesitancy reduced the likelihood of taking the Indian vaccine, and increased the odds of citing relative efficacy as a determinant of vaccine choice. This was despite the Indian vaccine being free, as cost considerations were also included in the Vaccine Hesitancy Index. Perhaps salient narratives around adverse effects following immunization (AEFI) may be activating the availability heuristic, rather than factual consideration of base rates of such effects being extremely rare. Beliefs in the effectiveness of COVID-19 vaccines in general also increased the odds of taking the Indian vaccine, with no effect on relying on the relative efficacy of Indian and foreign vaccines. This was in line with the health belief model, also applied in earlier work to determine the acceptance of COVID-19 vaccines among Indians (Goruntla et al., 2021). It is likely that individuals in the current sample were less concerned with the country of origin or price considerations of the vaccine, and more with any vaccination to safeguard against COVID-19. The role of informational factors influencing COVID-19 vaccine uptake is discussed in the following paragraph. Beliefs about effectiveness of mask-wearing lowered the odds of making this choice based on relative efficacy. Literature on non-pharmaceutical interventions suggests that stronger beliefs in mask-wearing may be prevalent when there is uncertainty on vaccine rollout (Lee et al., 2012) and point toward omitting concerns of relative efficacy when 
choosing to get vaccinated. Positive attitudes toward hand-washing increased the odds of considering the relative efficacy of vaccines prior to making a choice. Past evidence indicates that hand-washing is typically viewed as a habitual practice (Bish \& Michie, 2010) as well as a commonly accepted measure against respiratory illnesses (Teasdale et al., 2014). Thus, choosing a vaccine on the basis of relative efficacy may appeal to the "common sense" aspect associated with beliefs related to hand hygiene.

With respect to informational factors, choosing a vaccine based on relative efficacy increased when individuals were more likely to use newspapers, magazines, and the internet as primary sources of COVID-19 information (see also Murphy et al., 2021). Awareness in the form of availability of information is an important factor associated with vaccine uptake (Thomson et al., 2016) and individuals may rely on diverse information sources when deliberating about vaccination (see also Piltch-Loeb et al., 2021). Similarly, odds of choosing an Indian vaccine were lowered when individuals used these media outlets as their primary source of COVID-19 information; this could be because of the timing of the survey, as there were only preliminary reports on the efficacy of Covaxin (the Bharat Biotech vaccine) relative to Covishield (the AstraZeneca vaccine; Thiagarajan, 2021a). Individuals who reported that they had not been previously infected (but also not tested) were less likely to choose the Indian vaccine and more likely to rely on relative efficacy; this may also be an indication of lack of access to testing (Srinivasan, 2021), and potentially perceived lack of access to get vaccinated.

\section{Limitations and Future Research}

Although this study had merits, in that it was one of the first quantitative surveys to examine attitudinal and informational factors associated with vaccine uptake and choice in India, it had limitations. First, the period of data collection (Feb-March 2021) coincided with rapid 
changes in policies associated with the COVID-19 vaccine in India. For instance, the vaccine was rolled out to individuals over 60 years of age and those over 45 years with co-morbidities. Further, the cost of both Indian and foreign vaccines was capped at INR 250 (approx. USD 3); therefore, the hypothetical free Indian vaccine versus foreign vaccine in our survey may have yielded skewed responses. As data collection had begun before this directive, questions on actual vaccination behavior could not be included. Second, we were unable to account for attitudes as well as access to testing, as this could have influenced COVID-19 infection status and beliefs regarding vaccine uptake. Third, with respect to information sources, we did not include the nature of information accessed as well as belief in its veracity, both of which can impact vaccine attitudes and uptake. Finally, we are unable to explore the role of political beliefs and positioning on vaccine hesitancy and choice in the Indian context, which has been studied in other contexts (Hornsey et al., 2020).

In addition to addressing these limitations, future research can incorporate behavioral parameters, such as risk aversion and institutional trust, to examine vaccine hesitancy in the Indian context. Experimental work can focus on varying public health messaging related to the vaccine, keeping in mind the finding that belief in the effectiveness of masks is a robust predictor of vaccine choice and uptake. For example, there could be information-related field experiments that could determine what type and which mode of messaging works most effectively in boosting vaccine uptake in India. Further, messaging associated with the effectiveness of COVID-19 vaccines (in general) could have a salient impact on vaccine uptake in the Indian context. Working on developing such health communication is key to reducing vaccine hesitancy and improving vaccine coverage in the coming months in India. 
In conclusion, this study found that beliefs about vaccine effectiveness as well as its cost and side-effects are strongly associated with vaccine uptake in India. Furthermore, testing status, information sources, and vaccine hesitancy all predicted hypothetical choice of the vaccine.

\section{References}

Ajzen, I. (1991). The theory of planned behavior. Organizational Behavior and Human Decision Processes, 50(2), 179-211.

Andersson, O., Campos-Mercade, P., Meier, A. N., \& Wengström, E. (2021). Anticipation of COVID-19 vaccines reduces willingness to socially distance. Journal of Health Economics, 80, 102530. https://doi.org/10.1016/j.jhealeco.2021.102530

Asrani, P., Eapen, M. S., Hassan, M. I., \& Sohal, S. S. (2021). Implications of the second wave of COVID-19 in India. The Lancet Respiratory Medicine, 9(9), e93-e94. https://doi.org/10.1016/S2213-2600(21)00312-X

Bavel, J. J. V., Baicker, K., Boggio, P. S., Capraro, V., Cichocka, A., Cikara, M., Crockett, M. J., Crum, A. J., Douglas, K. M., Druckman, J. N., Drury, J., Dube, O., Ellemers, N., Finkel, E. J., Fowler, J. H., Gelfand, M., Han, S., Haslam, S. A., Jetten, J., ... Willer, R. (2020). Using social and behavioural science to support COVID-19 pandemic response. Nature Human Behaviour, 4(5), 460-471. https://doi.org/10.1038/s41562-020-0884-z

Bhuyan, A. (2021). India begins COVID-19 vaccination amid trial allegations. Lancet (London, England), 397(10271), 264. https://doi.org/10.1016/S0140-6736(21)00145-8

Bish, A., \& Michie, S. (2010). Demographic and attitudinal determinants of protective behaviours during a pandemic: A review. British Journal of Health Psychology, 15(4), 797824. https://doi.org/10.1348/135910710X485826 
Bloom, D. E., Fan, V. Y., \& Sevilla, J. P. (2018). The broad socioeconomic benefits of vaccination. Science Translational Medicine, 10(441), 1-4. https://doi.org/10.1126/scitranslmed.aaj2345

Brauer, M., Zhao, J. T., Bennitt, F. B., \& Stanaway, J. D. (2020). Global access to handwashing: Implications for COVID-19 control in low-income countries. MedRxiv, 1-6. https://doi.org/10.1101/2020.04.07.20057117

Chan, T. Y., Hamilton, B. H., \& Papageorge, N. W. (2016). Health, risky behaviour and the value of medical innovation for infectious disease. The Review of Economic Studies, 83(4), 1465-1510. https://doi.org/10.1093/restud/rdv053

Chevallier, C., Hacquin, A. S., \& Mercier, H. (2021). COVID-19 vaccine hesitancy: Shortening the last mile. Trends in Cognitive Sciences, 25(5), 331-333. https://doi.org/10.1016/j.tics.2021.02.002

Chou, W. Y. S., \& Budenz, A. (2020). Considering emotion in COVID-19 vaccine communication: Addressing vaccine hesitancy and fostering vaccine confidence. Health Communication, 35(14), 1718-1722. https://doi.org/10.1080/10410236.2020.1838096

Chowdhury, S. R., Motheram, A., \& Pramanik, S. (2021, April 14). Covid-19 vaccine hesitancy: Trends across states, over time. Ideas for India. https://www.ideasforindia.in/topics/humandevelopment/covid-19-vaccine-hesitancy-trends-across-states-over-time.html COVID-19 India dashboard. (2021). https://www.covid19india.org/

Danabal, K. G. M., Magesh, S. S., Saravanan, S., \& Gopichandran, V. (2021). Attitude towards COVID 19 vaccines and vaccine hesitancy in urban and rural communities in Tamil Nadu, India - a community based survey. BMC Health Services Research, 21(1), 994. https://doi.org/10.1186/s12913-021-07037-4 
Dash, R., Agrawal, A., Nagvekar, V., Lele, J., Di Pasquale, A., Kolhapure, S., \& Parikh, R. (2020). Towards adult vaccination in India: a narrative literature review. Human Vaccines and Immunotherapeutics, 16(4), 991-1001. https://doi.org/10.1080/21645515.2019.1682842

Goruntla, N., Chintamani, S., Bhanu, P., Samyuktha, S., Veerabhadrappa, K., Bhupalam, P., \& Ramaiah, J. (2021). Predictors of acceptance and willingness to pay for the COVID-19 vaccine in the general public of India: A health belief model approach. Asian Pacific Journal of Tropical Medicine, 14(4), 165. https://doi.org/10.4103/1995-7645.312512

Gudi, S. K., Chhabra, M., Undela, K., Venkataraman, R., Mateti, U. V., Tiwari, K. K., \& Nyamagoud, S. (2020). Knowledge and beliefs towards universal safety precautions during the coronavirus disease (COVID-19) pandemic among the Indian public: a web-based cross-sectional survey. Drugs and Therapy Perspectives, 36(9), 413-420. https://doi.org/10.1007/s40267-020-00752-8

Hornsey, M. J., Finlayson, M., Chatwood, G., \& Begeny, C. T. (2020). Donald Trump and vaccination: The effect of political identity, conspiracist ideation and presidential tweets on vaccine hesitancy. Journal of Experimental Social Psychology, 88(July). https://doi.org/10.1016/j.jesp.2019.103947

Jennings, W., Stoker, G., Willis, H., Valgardsson, V., Gaskell, J., Devine, D., Mckay, L., \& Mills, M. C. (2021). Lack of trust and social media echo chambers predict COVID-19 vaccine hesitancy. MedRxiv, 2021.01.26.21250246. http://medrxiv.org/content/early/2021/01/27/2021.01.26.21250246.abstract

Kanabar, K., \& Bhatt, N. (2021). Communication interventions to address COVID-19 vaccine hesitancy in India. Media Asia, 48(4), 330-337. 
https://doi.org/10.1080/01296612.2021.1965304

Kumari, A., Ranjan, P., Chopra, S., Kaur, D., Kaur, T., Kalanidhi, K. B., Goel, A., Singh, A., Baitha, U., Prakash, B., \& Vikram, N. K. (2021). What Indians think of the COVID-19 vaccine: A qualitative study comprising focus group discussions and thematic analysis. Diabetes and Metabolic Syndrome: Clinical Research and Reviews, 15(3), 679-682. https://doi.org/10.1016/j.dsx.2021.03.021

Lazarus, J. V., Ratzan, S. C., Palayew, A., Gostin, L. O., Larson, H. J., Rabin, K., Kimball, S., \& El-Mohandes, A. (2021). A global survey of potential acceptance of a COVID-19 vaccine. Nature Medicine, 27(2), 225-228. https://doi.org/10.1038/s41591-020-1124-9

Lee, C. K., Song, H. J., Bendle, L. J., Kim, M. J., \& Han, H. (2012). The impact of nonpharmaceutical interventions for $2009 \mathrm{H} 1 \mathrm{~N} 1$ influenza on travel intentions: A model of goal-directed behavior. Tourism Management, 33(1), 89-99. https://doi.org/10.1016/j.tourman.2011.02.006

Loomba, S., de Figueiredo, A., Piatek, S. J., de Graaf, K., \& Larson, H. J. (2021). Measuring the impact of COVID-19 vaccine misinformation on vaccination intent in the UK and USA. Nature Human Behaviour, 5(March). https://doi.org/10.1038/s41562-021-01056-1

Murphy, J., Vallières, F., Bentall, R. P., Shevlin, M., McBride, O., Hartman, T. K., McKay, R., Bennett, K., Mason, L., Gibson-Miller, J., Levita, L., Martinez, A. P., Stocks, T. V. A., Karatzias, T., \& Hyland, P. (2021). Psychological characteristics associated with COVID19 vaccine hesitancy and resistance in Ireland and the United Kingdom. Nature Communications, 12(1), 1-15. https://doi.org/10.1038/s41467-020-20226-9

Piltch-Loeb, R., Savoia, E., Goldberg, B., Hughes, B., Verhey, T., Kayyem, J., Miller-Idriss, C., \& Testa, M. A. (2021). Examining the effect of information channel on COVID-19 vaccine 
acceptance. MedRxiv, 1-27. https://doi.org/10.1101/2021.01.18.21250049

Ray, I. (2020). Viewpoint - Handwashing and COVID-19: Simple, right there...? World Development, 135(January), 105086. https://doi.org/10.1016/j.worlddev.2020.105086

Robinson, E., Jones, A., Lesser, I., \& Daly, M. (2021). International estimates of intended uptake and refusal of COVID-19 vaccines: A rapid systematic review and meta-analysis of large nationally representative samples. Vaccine, 39(15), 2024-2034. https://doi.org/10.1016/j.vaccine.2021.02.005

Sahoo, S., Padhy, S. K., Ipsita, J., Mehra, A., \& Grover, S. (2020). Demystifying the myths about COVID-19 infection and its societal importance. Asian Journal of Psychiatry, 54(January), 102244. https://doi.org/10.1016/j.ajp.2020.102244

Salali, G. D., \& Uysal, M. S. (2020). COVID-19 vaccine hesitancy is associated with beliefs on the origin of the novel coronavirus in the UK and Turkey. Psychological Medicine, 56-58. https://doi.org/10.1017/S0033291720004067

Sallam, M. (2021). Covid-19 vaccine hesitancy worldwide: A concise systematic review of vaccine acceptance rates. Vaccines, 9(2), 1-15. https://doi.org/10.3390/vaccines9020160

Sapkal, G. N., Yadav, P. D., Ella, R., Deshpande, G. R., Sahay, R. R., Gupta, N., Mohan, V. K., Abraham, P., Panda, S., \& Bhargava, B. (2021). Inactivated COVID-19 vaccine BBV152/COVAXIN effectively neutralizes recently emerged B 1.1.7 variant of SARSCoV-2. Journal of Travel Medicine. https://doi.org/10.1093/jtm/taab051

Schmid, P., Rauber, D., Betsch, C., Lidolt, G., \& Denker, M. L. (2017). Barriers of influenza vaccination intention and behavior - A systematic review of influenza vaccine hesitancy, 2005-2016. In PLoS ONE (Vol. 12, Issue 1). https://doi.org/10.1371/journal.pone.0170550

Schwarzinger, M., Watson, V., Arwidson, P., Alla, F., \& Luchini, S. (2021). COVID-19 vaccine 
hesitancy in a representative working-age population in France: a survey experiment based on vaccine characteristics. The Lancet Public Health, 6(April), 210-221. https://doi.org/10.1016/S2468-2667(21)00012-8

Srinivasan, S. (2021, April 7). Compulsory Covid-19 vaccination won't help - coercion will increase hesitancy, not reduce it. Scroll. https://scroll.in/article/991630/compulsory-covid19-vaccination-wont-help-coercion-will-increase-hesitancy-not-reduce-it

Tate, J., Aguado, T., Belie, J. De, Holt, D., Karafillakis, E., Larson, H. J., Nye, S., Salisbury, D., Votta, M., \& Wait, S. (2019). The life-course approach to vaccination: Harnessing the benefits of vaccination throughout life. Vaccine, 37(44), 6581-6583.

https://doi.org/10.1016/j.vaccine.2019.09.016

Teasdale, E., Santer, M., Geraghty, A. W. A., Little, P., \& Yardley, L. (2014). Public perceptions of non-pharmaceutical interventions for reducing transmission of respiratory infection: Systematic review and synthesis of qualitative studies. BMC Public Health, 14(1). https://doi.org/10.1186/1471-2458-14-589

Thiagarajan, K. (2021a). COVID-19: India is at centre of global vaccine manufacturing, but opacity threatens public trust. The BMJ, 372, 10-12. https://doi.org/10.1136/bmj.n196 Thiagarajan, K. (2021b). Why is India having a covid-19 surge? BMJ, April, n1124. https://doi.org/10.1136/bmj.n1124

Thomson, A., Robinson, K., \& Vallée-Tourangeau, G. (2016). The 5As: A practical taxonomy for the determinants of vaccine uptake. Vaccine, 34(8), 1018-1024. https://doi.org/10.1016/j.vaccine.2015.11.065

Tong, K. K., Chen, J. H., Yu, E. W. yat, \& Wu, A. M. S. (2020). Adherence to COVID-19 precautionary measures: Applying the health belief model and generalised social beliefs to a 
probability community sample. Applied Psychology: Health and Well-Being, 12(4), 12051223. https://doi.org/10.1111/aphw.12230

Wagner, A. L., Masters, N. B., Domek, G. J., Mathew, J. L., Sun, X., Asturias, E. J., Ren, J., Huang, Z., Contreras-Roldan, I. L., Gebremeskel, B., \& Boulton, M. L. (2019).

Comparisons of vaccine hesitancy across five low- and middle-income countries. Vaccines, 7(4), 1-11. https://doi.org/10.3390/vaccines7040155

World Health Organization. (2020). Survey tool and guidance: Behavioural insights on COVID19. In Survey tool and guidance: Behavioural insights on COVID. https://pesquisa.bvsalud.org/portal/resource/en/mdl-20203177951 


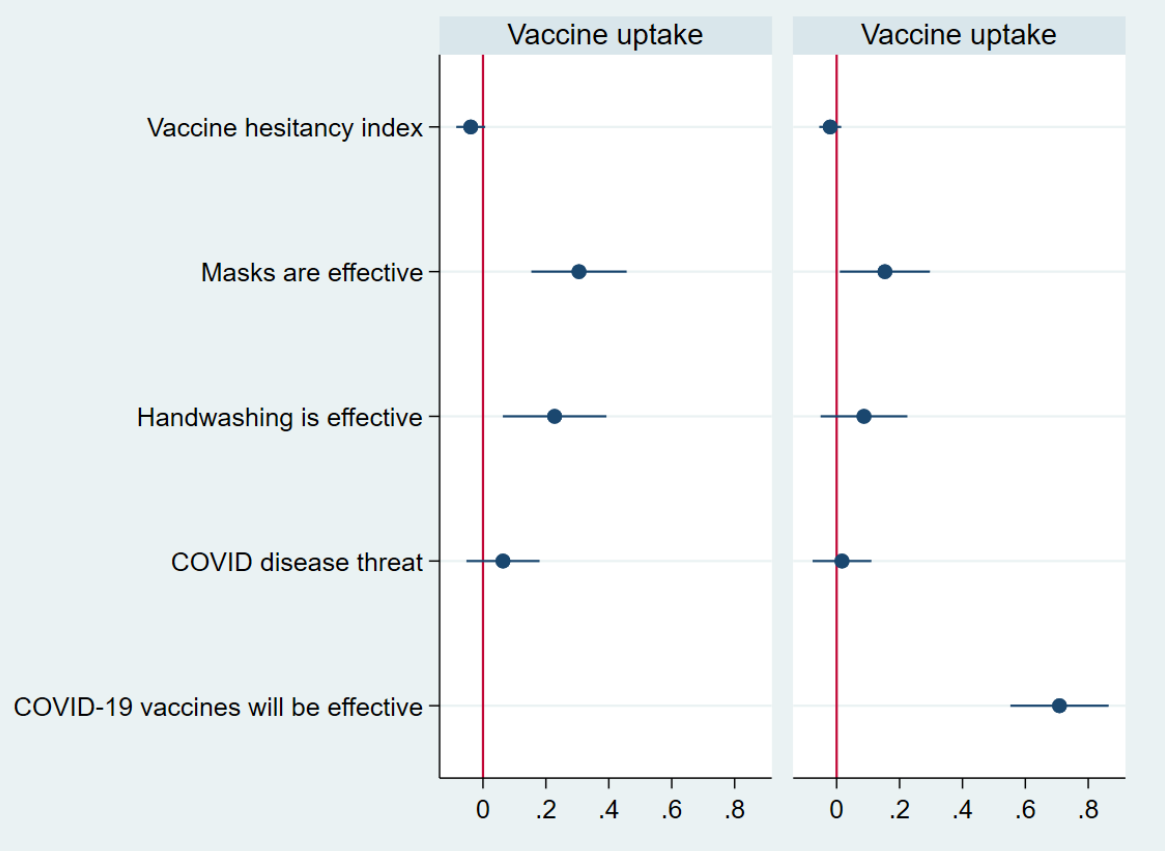

Figure 1a: COVID-19 beliefs and vaccine uptake

Note: Plot depicts point estimates of coefficients and $95 \%$ confidence intervals from two ordinary least squares regressions of vaccine uptake on vaccine hesitancy and other factors. The regression specification in the right panel is identical to the one on the left, except that it also includes an additional control variable for whether the respondent believes that COVID-19 vaccines will be effective. Regression results are provided in Table A. 2 in the appendix. 


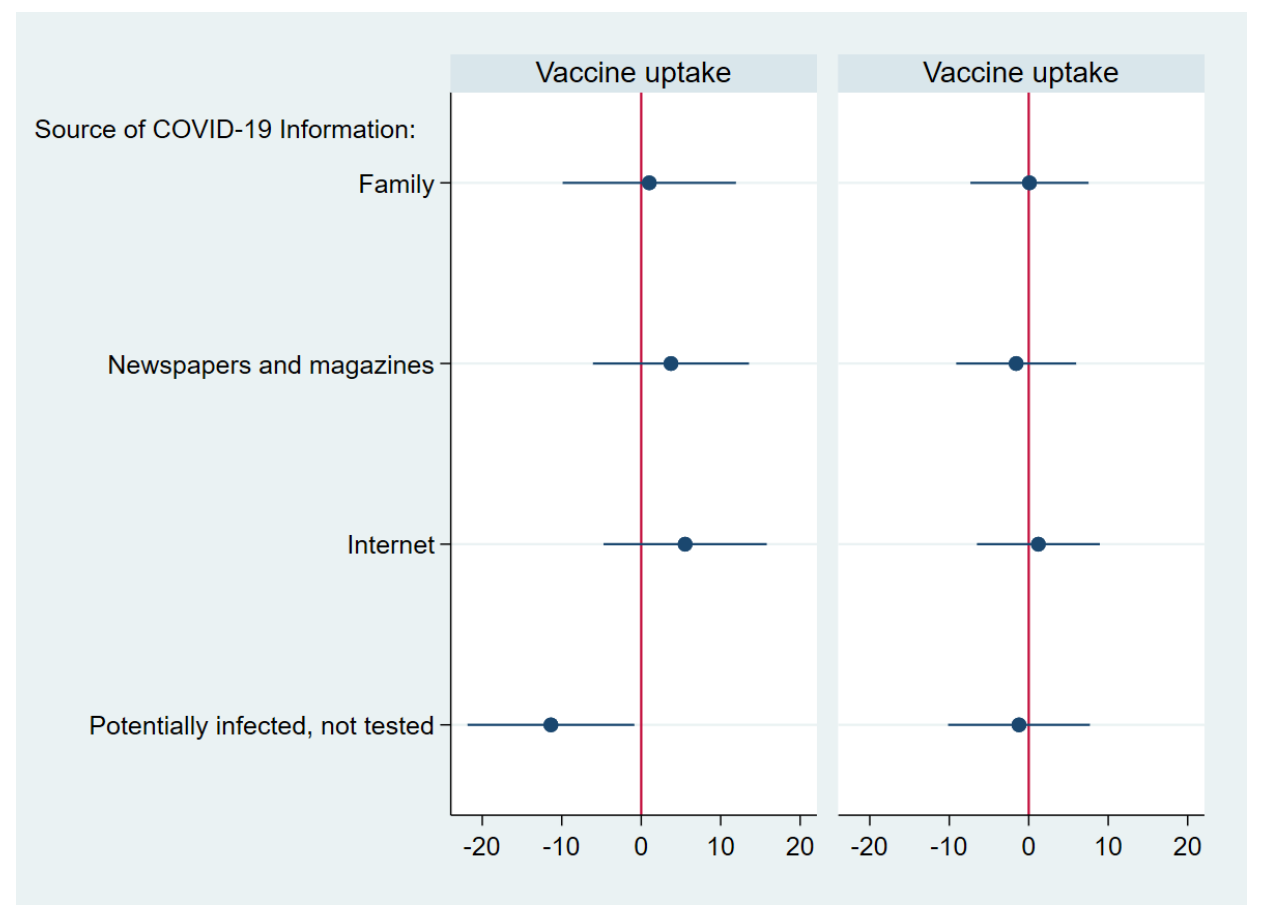

Figure 1b: COVID-19 information and testing on vaccine uptake

Note: Plot depicts point estimates of coefficients and 95\% confidence intervals from two ordinary least squares regressions of vaccine uptake on vaccine hesitancy and other factors. The regression specification in the right panel is identical to the one on the left, except that it also includes an additional control variable for whether the respondent believes that COVID-19 vaccines will be effective. Regression results are provided in Table A.2 in the appendix. 


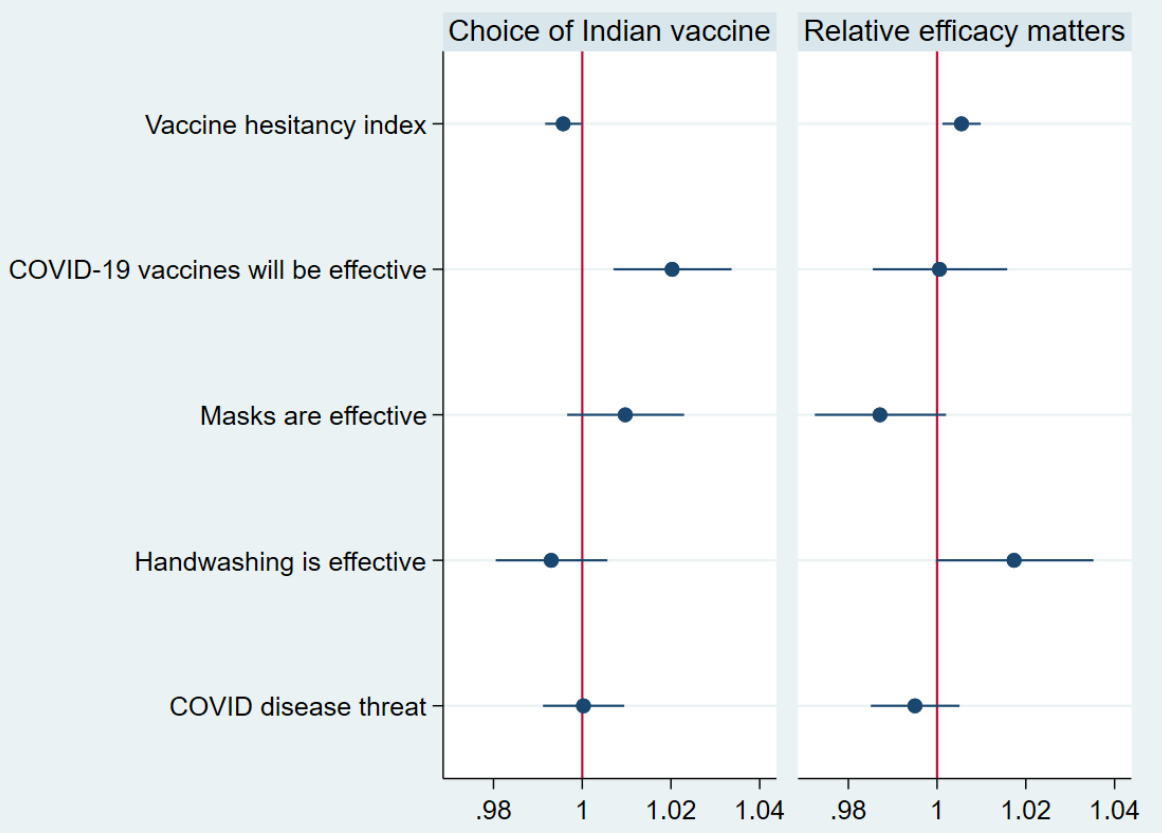

Figure 2a: COVID-19 beliefs and vaccine choice (odds ratios)

Note: Plot depicts point estimates of odds ratios and 95\% confidence intervals from two logistic regressions of hypothetical vaccine choice on vaccine hesitancy and other factors. Regression results are provided in Table A.2 in the appendix. 


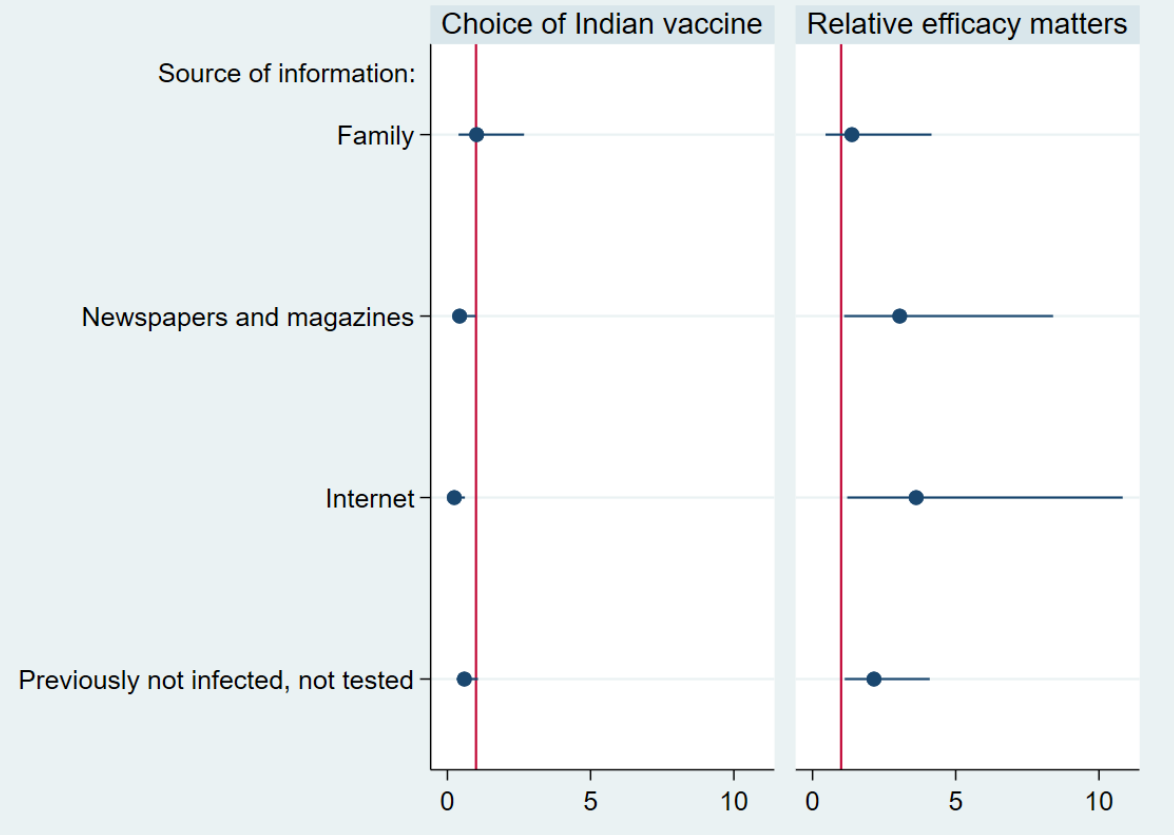

Figure 2b: COVID-19 information and testing on choice of vaccine (odds ratios)

Note: Plot depicts point estimates of odds ratios and 95\% confidence intervals from two logistic regressions of hypothetical vaccine choice on vaccine hesitancy and other factors. Regression results are provided in Table A.2 in the appendix. 